\title{
Attenuated Insular Processing During Risk Predicts Relapse in Early Abstinent Methamphetamine-Dependent Individuals
}

\author{
Joshua L Gowin', Katia M Harlé', Jennifer L Stewart', Marc Wittmann' ${ }^{1,2}$, Susan F Tapert ${ }^{1,3}$ and \\ Martin P Paulus ${ }^{1,3}$ \\ 'Psychiatry, UCSD, La Jolla, CA, USA; '2Empirical and Analytical Psychophysics, Institute for Frontier Areas of Psychology and Mental Health, \\ Freiburg, Germany; ${ }^{3}$ Psychiatry Service, VA San Diego Healthcare System, La Jolla, CA, USA
}

\begin{abstract}
There is some evidence that neuroimaging can be used to predict relapse among abstinent methamphetamine-dependent (MD) individuals. However, it remains unclear what cognitive and neural processes contribute to relapse. This investigation examined whether insula activation during risk-taking decisions - a process shown to be disrupted in MD—is able to predict susceptibility for relapse. Sixtyeight MD enrolled in a treatment program during early abstinence completed a risk-taking task during functional magnetic resonance imaging. Sixty-three of the sixty-eight individuals were followed up I year after the study. Of these, I 8 MD reported relapse. The 45 abstinent MD showed patterns of insula activation during risky decisions that resembled those found in prior studies of healthy controls, consisting of lower insula activation during safe decisions paired with higher activation during risky decisions. In contrast, the 18 relapsed MD showed similar insula activation during safe and risky decisions. An increase in one standard deviation in the difference in insula activation between risky and safe choices was associated with a 0.34 odds ratio for relapse at any given time. A median split of insula activation (difference between risky and safe) showed that individuals in the bottom half were two times more likely to relapse. In addition, a model that included several other brain regions increased prediction accuracy compared with insula-based model alone. These results suggest that failure to differentially activate the insula as a function of risk is a part of an altered risk-processing network associated with an increased susceptibility to relapse.

Neuropsychopharmacology (2014) 39, I379-1387; doi:I0.1038/npp.2013.333; published online 8 January 20I4
\end{abstract}

Keywords: risk-taking; decision-making; treatment; neuroimaging

\section{INTRODUCTION}

A significant number of individuals with methamphetamine dependence who enter a treatment program will relapse in the year following treatment (McKetin et al, 2012; Miller, 1996). Identifying individuals at greatest risk for relapse could allow more targeted treatment; individuals at risk may need more intense interventions, more frequent interaction with a health-care provider, or to be alerted about their risk. Recent studies have focused on developing neuroimaging as a tool to predict which individuals will relapse (Kosten et al, 2006; Paulus et al, 2005). Several studies have shown that stimulant-dependent and alcoholdependent individuals who were more likely to relapse exhibited less activation in the insular and anterior cingulate cortices within the context of a variety of tasks, including error detection, drug-cue detection, and stress processing (Clark et al, 2012; Luo et al, 2013; Marhe et al,

*Correspondence: Dr JL Gowin, Psychiatry, University of California San Diego, 8939 Villa La Jolla Dr Suite 200, La Jolla, CA 92037, USA, Tel: + I 97039660 II, Fax: + I 8588223933 ,

E-mail: joshua.gowin@gmail.com

Received 22 August 2013; revised 19 November 2013; accepted 25 November 2013; accepted article preview online 6 December 2013
2013; Seo et al, 2013). However, no study has focused on the neural correlates of risk-taking as a predictor of relapse despite evidence that substance use disorders are associated with altered neural processing of risk (Cousijn et al, 2013; Gowin et al, 2013).

Behavioral evidence suggests that methamphetaminedependent (MD) individuals take more risks than comparison participants, and neuroimaging studies show evidence for altered processing of risk among MD (Gowin et al, 2013). Economists define risk as the selection among options with variably distributed outcomes (Kahneman and Tversky, 1979). This definition differs from the broader meaning of risk used by clinicians and the lay population, which also involves uncertainty, but emphasizes the possibility of loss associated with a rewarding option (Schonberg et al, 2011). Studies with healthy volunteers suggest that the anterior cingulate cortex and the insula are involved in the processing of risky decisions (Brown and Braver, 2007; Paulus et al, 2003; Preuschoff et al, 2008; Rudorf et al, 2012). The insula may be important for decisions under risk because of its purported role in anticipating how a decision will make the individual feel (Craig, 2009; Simmons et al, 2009). For example, healthy volunteers with higher levels of harm avoidance showed higher levels of insula activation as they prepared to make risky choices, suggesting that the 
insula may be involved in the anticipation of future states (Paulus et al, 2003). Several studies suggest that activation in the anterior cingulate cortex and the insula may be disrupted in substance-dependent individuals, with some indication that stimulant-dependent individuals show decreased activation relative to healthy groups (Ersche et al, 2005; Fishbein et al, 2005; Kaufman et al, 2003; Kim et al, 2011; Nestor et al, 2011). It remains unclear whether individuals who relapse process risk differently from individuals who remain abstinent or whether potential differences in risk processing might contribute to relapse likelihood.

The present study investigated whether activation in the insula and the anterior cingulate cortex during risk-taking would distinguish $\mathrm{MD}$ who relapse from MD who maintain abstinence. We hypothesized that MD who maintained abstinence would show activation patterns resembling healthy individuals, with increased activation in proportion to increased risk signaling risk aversion (Paulus et al, 2003). Support for this hypothesis would provide evidence for a process-driven dysfunction that has practical consequences over the course of methamphetamine dependence. Riskprocessing deficits may provide an approach for future interventions that target specific neural processes.

\section{MATERIALS AND METHODS}

\section{Participants}

The study protocol was approved by the UCSD Human Research Protections Program and carried out in accordance with the Declaration of Helsinki. Sixty-eight (fifteen female) MD were recruited through 28-day inpatient Alcohol and Drug Treatment Programs at the Veterans Affairs San Diego Healthcare System and Scripps Green Hospital (La Jolla, CA). To maintain sobriety during the program, participants were screened for the presence of drugs via urine toxicology. Study procedures occurred during the third or fourth week of treatment. All participants had ceased using methamphetamine for an average of $34.0 \pm 3.4$ days before study procedures (range of 15-207 days).

Lifetime DSM-IV (American Psychiatric Association, 1994) Axis I diagnoses (including substance dependence) and Axis II antisocial personality disorder were assessed by experienced interviewers using the Semi-Structured Assessment for the Genetics of Alcoholism (SSAGA) (Hesselbrock et al, 1999). Diagnoses were based on consensus meetings with a clinician specialized in substance use disorders (MPP) and trained study personnel. Exclusion criteria included: (1) antisocial personality disorder; (2) current (past 6 months) Axis I panic disorder, social phobia, posttraumatic stress disorder, major depressive disorder; (3) lifetime bipolar disorder, schizophrenia, and obsessive compulsive disorder; (4) current severe medical disorders requiring in-patient treatment or frequent medical visits; (5) use of medications that affect the hemodynamic response within the past 30 days such as antihypertensives, insulin, and thyroid medication; (6) current positive urine toxicology test; and (7) history of head injuries with loss of consciousness for longer than $5 \mathrm{~min}$. During evaluation, participants performed the North American Adult Reading Test (NAART; Uttl, 2002) as a measure of verbal intelligence (VIQ). Characteristics are summarized in Table 1.
Table I Subject Characteristics by Group

\begin{tabular}{|c|c|c|c|c|c|}
\hline \multirow[t]{2}{*}{ Characteristic } & \multicolumn{3}{|c|}{ Abstinent } & \multicolumn{2}{|c|}{ Relapsed } \\
\hline & $\mathbf{N}$ & & $\%$ & \multirow{2}{*}{$\frac{N}{18}$} & $\%$ \\
\hline Participants & 45 & & & & \\
\hline Female & 11 & & 23 & 4 & 21 \\
\hline White & 24 & & 51 & 12 & 63 \\
\hline Black & 5 & & 11 & 3 & 16 \\
\hline Hispanic & 17 & & 35 & 4 & 21 \\
\hline Asian & । & & 2 & 2 & II \\
\hline Alcohol dependence ${ }^{\mathrm{a}}$ & 18 & & 37 & 10 & 53 \\
\hline Marijuana dependence ${ }^{a}$ & 8 & & 17 & 5 & 26 \\
\hline Cocaine dependence $^{a}$ & 13 & & 27 & 7 & 37 \\
\hline Cocaine dependence ${ }^{b}$ & 5 & & 10 & 4 & 21 \\
\hline Alcohol dependence ${ }^{b}$ & 8 & & 17 & 6 & 32 \\
\hline \multirow[t]{2}{*}{ Marijuana dependence $e^{b, c}$} & 2 & & 4 & 4 & 21 \\
\hline & & Mean & SD & Mean & SD \\
\hline Age (years) & & 38.8 & II.I & 37.4 & 9.2 \\
\hline Education (years) & & 12.8 & 1.7 & 13.3 & 1.5 \\
\hline Verbal $I Q^{d}$ & & 108.0 & 10.2 & 109.7 & 7.3 \\
\hline Alcohol (drinks/week) & & 11.0 & 17.6 & 14.4 & 33.0 \\
\hline Nicotine (cigarettes/day) ${ }^{e}$ & & 11.8 & 9.3 & 8.7 & 9.2 \\
\hline $\begin{array}{l}\text { Methamphetamine estimated } \\
\text { lifetime uses }\end{array}$ & & 14624.5 & $324 \mid 4.1$ & 8841.6 & 12353.1 \\
\hline Methamphetamine age onset & & 24.1 & 9.4 & 24.9 & 9.1 \\
\hline Methamphetamine recent use (da) & & 35.6 & 33.2 & 31.5 & 16.7 \\
\hline Cocaine $e^{f, g}$ & & 2551.4 & 6116.9 & 3942.9 & 7250.8 \\
\hline Marijuana $^{f}$ & & 10882.5 & 30375.4 & 4743.5 & 8851.2 \\
\hline \multicolumn{6}{|c|}{$\begin{array}{l}\text { aMet criteria for lifetime comorbid dependence with methamphetamine } \\
\text { dependence. }\end{array}$} \\
\hline \multicolumn{6}{|c|}{$\begin{array}{l}{ }^{b} \text { Met criteria for current comorbid dependence with methamphetamine } \\
\text { dependence. } \\
c^{c}<0.05 \text { based on chi-squared analysis. } \\
{ }^{d} \text { Assessed by the North American Adult Reading Test (NAART; Uttl, 2002) }\end{array}$} \\
\hline
\end{tabular}

All subjects gave written informed consent to participate in a clinical interview session, a functional magnetic resonance imaging (fMRI) session, and a follow-up phone interview 1 year later to assess abstinence. SSAGA interviews revealed that no subjects were experiencing symptoms of withdrawal before neuroimaging. Follow-up consisted of a structured interview based on the SSAGA. Participants were asked about their current living and working situation and whether they had used any of the following substances in the past year: sedatives, hallucinogens, stimulants, marijuana, or opiates. Relapse was defined as any use of these substances. On the basis of responses, $45 \mathrm{MD}$ (11 female) remained abstinent from drugs (with the exception of nicotine) from the time of treatment to follow-up (abstinent group), $18 \mathrm{MD}$ (4 female) relapsed and 5 participants could not be tracked at follow-up. 


\section{Risky Gains Task}

The Risky Gains Task (RGT) has been used in a number of risk-taking studies (Kruschwitz et al, 2012; Paulus et al, 2003) and is briefly described here. Subjects were told that the goal of the RGT was to earn as many points as possible. Points were exchanged for money when the task ended. Participants earned points (as value in cents) by selecting, with a button press, one of three options - 20, 40, or 80 -on each trial. The options appeared in ascending order for $1 \mathrm{~s}$ each. Participants were told 20 was the safe option and 40 and 80 were risky options. If a participant pressed the button when 20 appeared, then the trial ended and the subject received 20 points. If the participant bypassed 20, then the 40 option appeared. On some trials, the participant immediately lost 40 points and the trial ended. Otherwise, the subject had the option to press the button and earn 40 points. If 40 was bypassed, then 80 appeared. Like the 40 option, sometimes the participant immediately lost 80 points and the trial ended. Otherwise, the participant pressed the button, received 80 points and the trial ended. The task consisted of 96 trials, each lasting $3.5 \mathrm{~s}$ regardless of the participant's response. The three trial types were presented in a predetermined, randomized order: 54 unpunished $(+20,+40,+80), 24$ punished $(-40)$, and 18 punished $(-80)$. If a participant selected 20 on a -40 trial, then they received 20 points. Similarly, if they selected 20 or 40 on a -80 trial, then they received 20 or 40 points, respectively. Unbeknownst to participants, the number of negative trials for 40 and 80 options ( -40 and -80$)$ was set so that choosing the same option on each trial would produce the same final points total, that is, choosing risky or safe resulted in the same point total.

\section{Image Acquisition}

A fMRI run sensitive to blood-oxygenation level-dependent (BOLD) contrast was collected using a Signa EXCITE 3T scanner (GE Healthcare, Milwaukee, Wisconsin, T2*-weighted echoplanar imaging; $\mathrm{TR}=2000 \mathrm{~ms}, \mathrm{TE}=32 \mathrm{~ms}, \mathrm{FoV}=230$ $\times 230 \mathrm{~mm}^{2}, 64 \times 64$ matrix, $302.6-\mathrm{mm}$ axial slices with $1.4 \mathrm{~mm}$ gap, flip angle $=90^{\circ}$, total duration: $8 \mathrm{~min}, 32 \mathrm{~s}$, $3.59 \times 3.59 \times 2.6 \mathrm{~mm}^{3}$ voxels). Six resting trials were collected at preset points during the task, but were excluded from analysis. For anatomical reference, a high-resolution, T1 weighted image $(\mathrm{TR}=8 \mathrm{~ms}, \mathrm{TE}=3 \mathrm{~ms}, \mathrm{FoV}=250 \times$ $250 \mathrm{~mm}^{2}, \quad 192 \times 256$ matrix interpolated to a $256 \times 256$ matrix, flip angle $=12^{\circ}, 172$ sagitally acquired slices, $.97 \times$ $97 \times 1 \mathrm{~mm}^{3}$ voxels) was collected during the same session.

\section{Behavioral Analysis}

In all, 40 and 80 choices were combined as a single metric of risk-taking. Frequency of risky choices was compared overall (across all trials) and following a loss (loss on the previous trial). A two-way repeated measures analysis of variance (ANOVA) examined frequency of risky choices to test for a main effect of group (ie, relapse $v s$ abstinent), condition (ie, overall vs post-loss), and a group-bycondition interaction.

\section{fMRI Preprocessing}

Data were preprocessed using the Analysis of Functional NeuroImages (AFNI) software (Cox, 1996). Echoplanar images were aligned to anatomical images. Outlier voxels were identified in the aligned images based on whether a given time point greatly exceeded the mean number of voxel outliers for the time series; time points with high numbers of outlier voxels were excluded from subsequent analyses. Images were spatially smoothed using a 4-mm Gaussian filter, normalized to Talairach space and visually inspected. Preprocessed data were analyzed using a multiple regression model based on a BOLD response function with a 4- to 6-s peak.

\section{Primary Analytic Strategy}

A general linear model fit was performed using AFNI's $3 \mathrm{dDeconvolve}$ function, wherein regressors for safe $(+20)$ and risky $(+40,+80)$ decisions were defined as starting at trial onset and concluding when the subject made (1) a response or (2) a punishment $(-40,-80)$ was delivered. Baseline activation was calculated from the BOLD signal during intertrial intervals and six null trials (ie, fixation on crosshairs without responding) that were interleaved into the RGT. Three motion regressors (roll, pitch, and yaw) and a regressor for slow linear drift were included in the linear model fit as regressors of non-interest. Percent signal change (PSC) was calculated by dividing the regressor of interest by the baseline regressor.

To test the hypothesis that relapsed MD would process risk differently as a function of insula activation, a linear mixed-effects (LME) analysis was conducted using the $\mathrm{R}$ statistical software (www.r-project.org). Group (relapse and abstinent) and decision (safe and risky) were fixed effects in the model and individual participants were treated as random effects. LME analysis examined the main effect of group and the group-by-decision (ie, risky $v s$ safe) interaction. Analyses were performed voxel-wise but restricted to a mask that included the bilateral insula and the anterior cingulate cortex based on a priori hypotheses. A volume threshold adjustment for a significant activation was performed based on the results of AFNI's AlphaSim program to prevent typeI errors (two-tailed $p<0.05$ cluster size thresholds: bilateral insula: $320 \mu \mathrm{l}$, or 5 contiguous voxels; bilateral anterior cingulate: $384 \mu \mathrm{l}$, or 6 contiguous voxels). A whole brain analysis of group differences is presented in Supplementary Figure 1. For each significant activation cluster identified in this analysis, center-of-mass coordinates are reported in Talairach space $(\mathrm{x}, \mathrm{y}, \mathrm{z})$ and labeled using the Talairach Daemon software (Lancaster et al, 2000).

\section{Secondary Analytic Strategy}

To explore the implications of the LME analysis, we correlated risk-taking behavior during the task with the differential activation between risky and safe decisions in the clusters identified by the LME across both groups. This analysis aimed to show that brain activation was related to behavior and not an artifact.

To determine whether the risk-related insular activation was related to MD's time to relapse, a Cox proportional 
hazards regression was used. Cox regression provides a way to test whether variables relate to the likelihood of an event (eg, relapse to methamphetamine use) when not all participants have experienced the event. The model computes a coefficient for each independent variable. Assuming two individuals are the same for all other variables, the exponent of the coefficient represents the hazards ratio between the two individuals per unit of time. This can also be interpreted as the instantaneous relative risk of an event (Rosner, 2006).

Two Cox models were computed. First, independent variables included clinical (days since last use of methamphetamine, estimated total lifetime uses of methamphetamine) and behavioral variables (risky behavior during the task). Second, the first model was augmented with insula activation (difference between average right insula activation during risky and safe decisions extracted from areas identified in group-by-decision LME differences, ie, riskysafe $=$ differential activation). Right insula activation was chosen exclusively because of the high collinearity with left insula activation $(r=0.68)$ and because a previous study in MD individuals showed that right insula activation predicted relapse (Paulus et al, 2005). All independent variables were z-transformed before analysis so that the exponent of model coefficients would equal the odds ratio that a change in one standard deviation would result in relapse/survival. To examine the predictive value of each independent variable, receiver operating characteristic (ROC) curves were calculated. For ROC curves, lines that hew closely to the diagonal line offer no better predictive value than chance. The further above the diagonal is, the more specificity or sensitivity the test offers. Thus, an area under the curve (AUC) close to 0.5 offers little predictive value, but as AUC approaches 1, predictions improve. A Bayes Nomogram was drawn to visualize odds ratio and relative risk for insula activation (risky-safe) based on a median split where MD in the bottom half were predicted to relapse.

\section{RESULTS}

\section{Demographics}

After 1 year, 29\% $(N=18)$ of the sample relapsed (see Figure 1). Relapse and abstinent MD were similar in age, education, and verbal IQ (all $p>0.10$, see Table 1 ). They had used similar amounts of methamphetamine across the lifetime $(t=1.24, p>0.10)$. There were no significant differences in comorbid dependence on alcohol or cocaine $(p>0.05)$. Relapse rates did not differ between males and females $(p>0.05)$. Both groups smoked similar amounts of cigarettes and marijuana and drank similar amounts of alcohol (all $p>0.10$ ). There was a trend for relapse MD to have used more cocaine across the lifetime than abstinent $\mathrm{MD}(t=1.85, p=0.07)$.

\section{Behavioral Data}

To determine whether risk-taking behavior varied between groups, a two-way, repeated-measures ANOVA was conducted. There was no main effect of group $\left(\mathrm{F}_{1,61}=0.09\right.$, $p=0.76)$ or condition $\left(\mathrm{F}_{1,61}=0.52, p=0.47\right)$. There was no group-by-condition interaction $\left(\mathrm{F}_{1,61}=0.003, p=0.96\right)$.

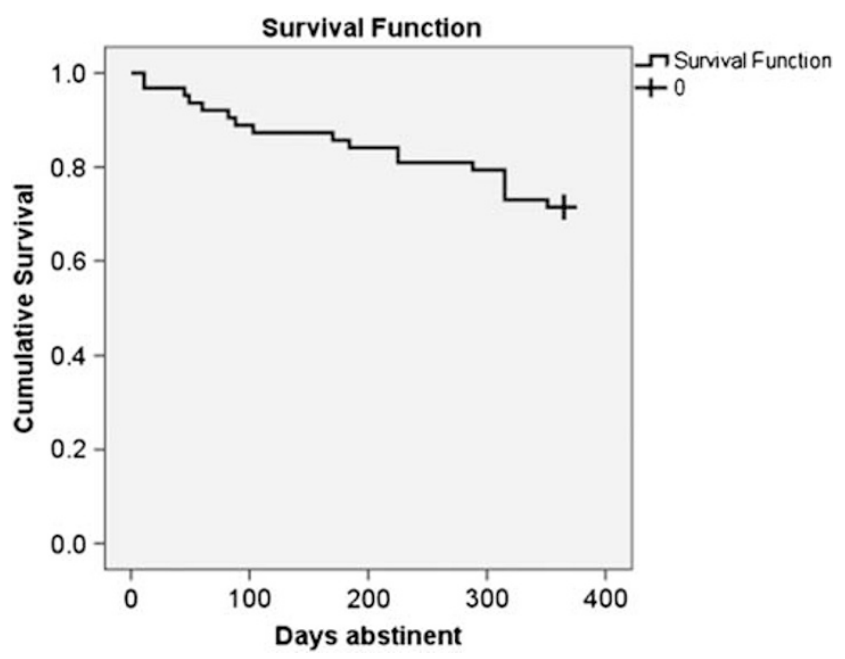

Figure I The survival curve bends at each point that a participant relapsed in the year after treatment. The participants who survived are represented by the crosshair at the right side of the curve.

\section{Imaging Data}

Group main effect. There were no clusters within the mask that showed a main effect of group.

Group-by-decision interaction. LME analysis indicated a significant group-by-decision interactions in bilateral anterior insula (BA 13; right cluster: $x=34, y=9, z=14$, Vol. $=832$ $\mu \mathrm{l}$; left clusters: $x=-36, y=13, z=12$, Vol. $=640 \mu \mathrm{l}$ and $x=-36, y=2, z=2$, Vol. $=576 \mu \mathrm{l})$. Specifically, whereas abstinent MD showed less activation during safe decisions and increased activation during risky decisions, relapse MD showed no difference between safe and risky decisions (see Figure 2). No regions of the anterior cingulate cortex had significant group-by-decision effects. Whole brain analysis revealed significant group-by-decision interactions in several other regions of the brain, including the dorsolateral prefrontal cortex, thalamus, dorsal striatum, middle temporal gyrus, posterior cingulate, and ventromedial prefrontal cortex (see Supplementary Figure 1 and Supplementary Table 1).

Brain-behavior relationship. Correlation analyses revealed a significant relationship between bilateral anterior insula activation and risk-taking behavior (see Figure 2). MD with greater activation during a risky relative to a safe decision took fewer risks overall (right cluster: $r=-0.29, p=0.02$; left cluster: $r=-0.30, p=0.02$ ).

\section{Cox Proportional Hazards Model}

As seen in Table 2, the overall model was not significant in predicting risk for relapse when only behavioral and clinical variables were included $\left(-2 \log\right.$ likelihood $=141.8, \chi^{2}(3)=$ $2.3, p<0.52)$. However, when the model included right insula activation, the model became significant $(-2 \log$ likelihood $\left.=128.9, \chi^{2}(3)=12.6, p=0.01\right)$. Higher levels of right insula activation during a risky relative to a safe 

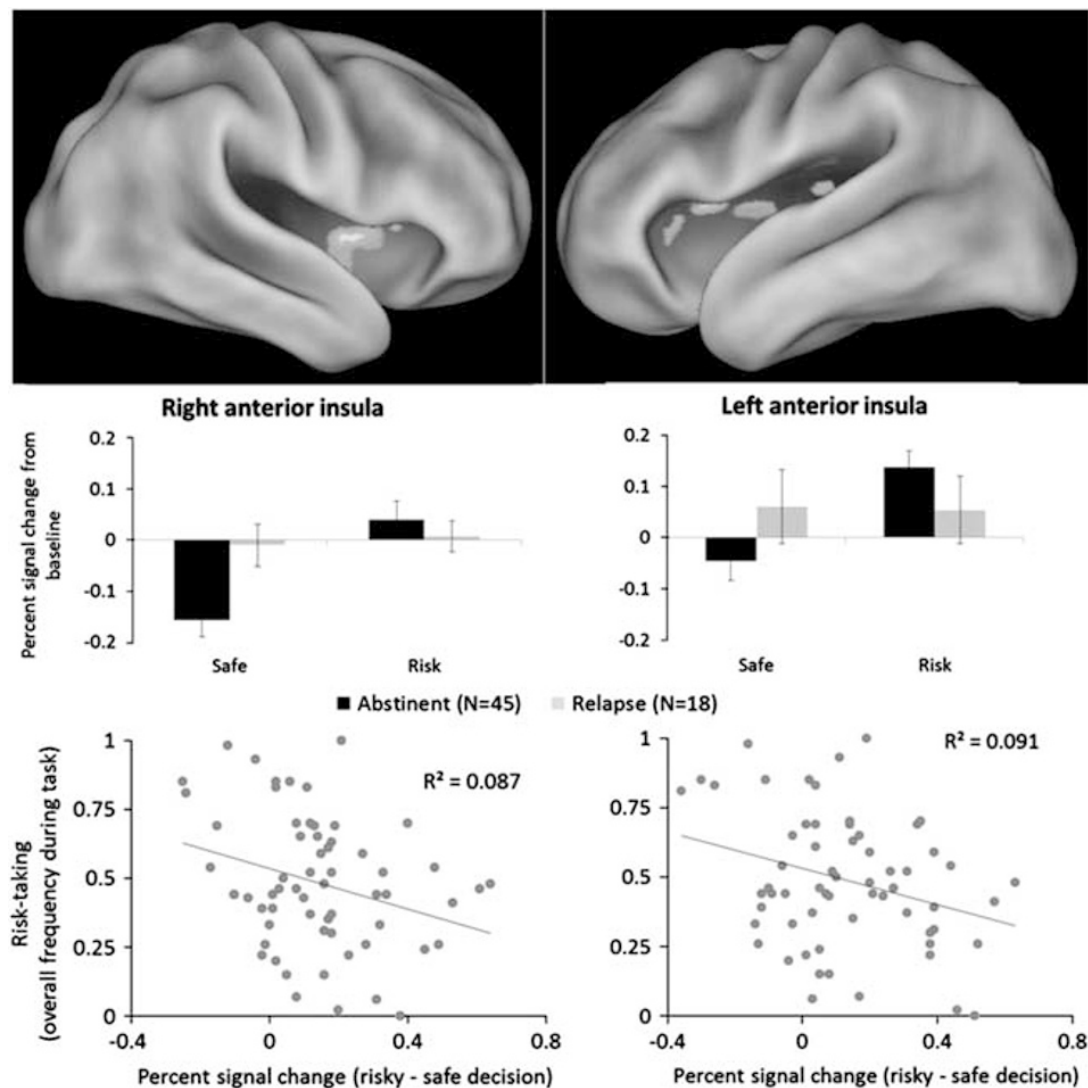

Figure 2 Upper panel: Group-by-decision interaction. The abstinent group showed less activation in the bilateral anterior insula during safe decisions but increased activation during risky decisions. The relapse group showed no change in insular activation between risky and safe decisions. Insular activation related to risk processing among the abstinent group mirrored healthy individuals, where activation increases in proportion to risk. Error bars represent standard error. Lower panel: The differential activation between risky and safe decisions (risky-safe) was significantly correlated with risk-taking frequency for both the left and right clusters identified in the LME model shown above, indicating that insular activation during decisions was related to task behavior.

decision $(B=-1.1 \pm .32, p=0.001)$ predicted a 2.9 times lower likelihood of relapse, supporting our hypothesis that greater insula activation during a risky relative to a safe decision may help individuals abstain from methamphetamine use.

We examined the predictive value of each region and included a Cox regression with clusters in the right posterior cingulate, the right medial frontal gyrus, the left thalamus, the left ventromedial prefrontal cortex, and the left lentiform nucleus. This model was predictive of relapse and added variance explained compared with the insula alone. In this model, the insula no longer independently predicted relapse, which is likely due to the high collinearity of the variables in the model, presented in Supplementary Table 4

\section{Predictive Value of the Model}

As seen in Figure 3, right insula activation provided higher sensitivity and specificity than clinical or behavioral variables. The AUC was 0.76 for the insula, 0.60 for lifetime methamphetamine use, and 0.53 for risk-taking behavior. The sample was divided into two groups based on a median split of right insula activation. On the basis of this classification, 14 of $18 \mathrm{MD}$ were correctly identified as relapsing (specificity $=0.60$ ) and 27 of $45 \mathrm{MD}$ were correctly identified as maintaining abstinence (sensitivity $=0.78$ ). As seen in the Bayes Nomogram in Figure 3, an individual in the bottom half of insula activation was twice as likely to relapse in the year following treatment. In contrast, an individual in the top half of insula activation was 2.5 times less likely to relapse in the year following treatment.

\section{DISCUSSION}

This investigation tested the hypothesis that attenuated neural processing of risky decisions is predictive of which treatment-seeking MD relapse in the year after treatment. Indeed, those who relapsed showed blunted risk-related processing in insular cortex compared with those who stayed abstinent. Since neither clinical nor behavioral characteristics differed between groups, differences in neural activation during risk-taking may offer unique predictive information regarding relapse likelihood. On the basis of evidence that insula activation increases in proportion to risk (Paulus et al, 2003; Preuschoff et al, 2008; Rudorf et al, 2012), individuals who show deviations from this pattern, potentially indicating disrupted risk processing, may be at greatest risk for relapse. Furthermore, a set 
Table 2 The First Step of the Cox Model Assesses the Predictive Value of Lifetime History of Methamphetamine use (total uses), Days Since Last Use and Risk-taking Behavior

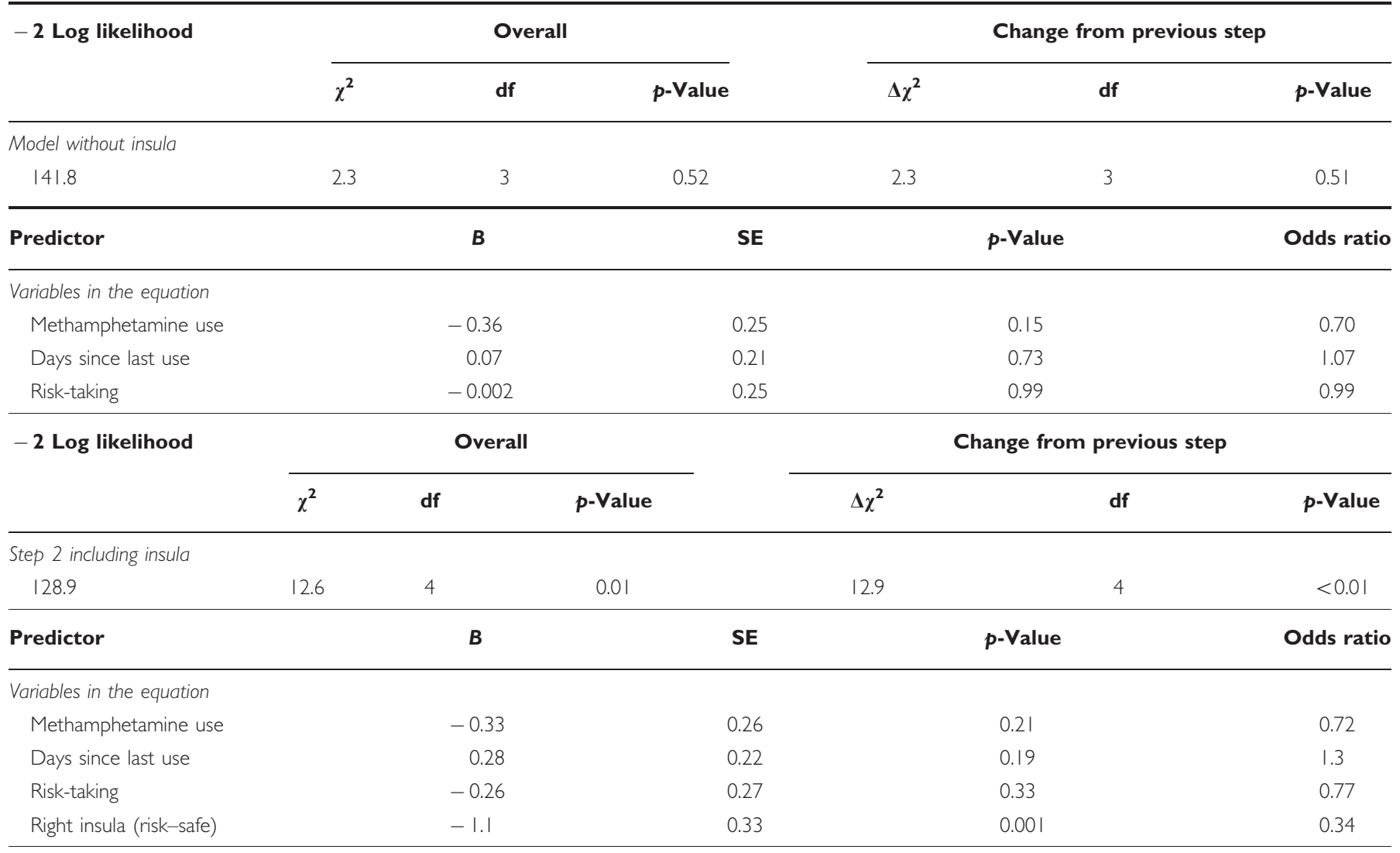

In the second step, right insula is added. Right insula represents average activation during a risky decision minus activation during a safe decision, reflecting the differential activation between risk and safe.

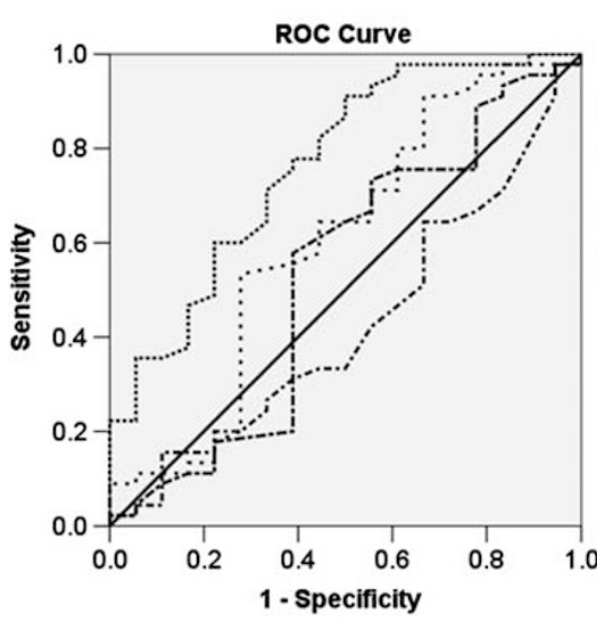

Source of the Curve .... Right insula Lifetime .. methamphetamine uses

... Days since last

use

-.. Risk-taking

- Reference Line

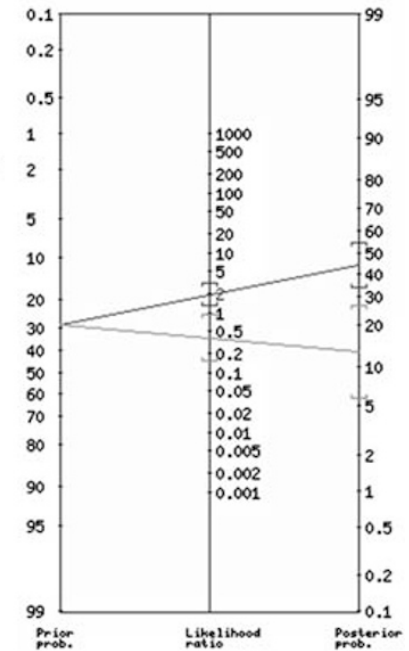

Figure 3 The receiver operating characteristic (ROC) curves on the left show that right insula activation provides more sensitivity and specificity than total lifetime uses of methamphetamine, days since last use of methamphetamine or risk-taking during the behavioral task. The Bayes Nomogram on the right shows that a median split of insula activation during decision-making (risk-safe activation) provides improved predictive value relative to the prior probability of 0.29 for relapse. Individuals in the bottom half of insula activation had a 0.45 probability of relapse whereas those in the top half had only a 0.12 probability of relapse. 
of regions implicated in risky decision-making added variance explained in predicting relapse, suggesting that a network of regions may be involved in risk-taking that leads to relapse. Since MD had co-morbid use of substances including cocaine, marijuana, and alcohol, these results may not simply be due to the consequences of MD but may also extend to other drugs. For reasons we outline below, the lack of differential activation in the insula during risky $v s$ safe choices, even in the absence of behavioral differences, may indicate that these individuals experience potential risk differently and this may set the stage for decisions leading to relapse.

First, the insular cortex, particularly the anterior portion, has been suggested to have a role in interoception, processing, and conscious awareness of internal feelings (Craig, 2009). Psychological research has demonstrated that feelings influence how humans perceive risk as much as deliberate calculations of probability (eg, 'I feel scared when I fly in airplanes so I think it's risky form of transportation, even though I know car crashes are much more frequent') (Slovic et al, 2002). Given its role in interoception, the anterior insula is a plausible neural substrate involved in risk perception via positive or negative feelings. This is supported by neuroimaging work showing that insula activation corresponds to the anticipation of negative experiences (Paulus et al, 2003). For instance, in similar risk-taking paradigms, healthy individuals with greater insula activation during the decision phase of the task are more likely to avoid risky options and have higher levels of harm avoidance (Paulus et al, 2003; Rudorf et al, 2012). The relationship between insula activation and risk aversion may reflect fear of loss. Further, anterior insular activation to choice options has been shown to predict more riskaverse decisions in financial investment paradigms (Kuhnen and Knutson, 2005) and to mediate higher rejection of ultimatum bargaining due to negative affect (Harle et al, 2012). These studies suggest that the insula has a role in facilitating decision adjustments based on affective signals such as risk anticipation. This may be useful for making decisions to avoid risky, potentially harmful situations.

Second, there is evidence that MD demonstrate emotionprocessing deficits, including appraisal of negative affective cues, which may affect their decision-making. For instance, MD perform worse and show reduced activation in the insula in affect-matching paradigms (Kim et al, 2011; Nestor et al, 2011; Payer et al, 2008). This is consistent with MD's relative insensitivity to the negative consequences of drug abuse (Koob, 2008; Li and Sinha, 2008). This research suggests that MD may have disrupted processing of feeling states that disrupt their decision-making in a variety of contexts, such as risk-taking. On the basis of our results, such deficits may specifically underlie vulnerability to relapse. The present results suggest that MD who relapsed in the year following treatment fail to show differential insula activation between risky and safe options during the decision phase of a risk-taking task, suggesting that they may fail to appropriately anticipate the likelihood or magnitude of a negative event. Blunted neural processing of risk could make them more likely to enter situations that increase relapse likelihood (eg, spending more time with drug-using friends, underestimating negative health consequences of drug use) because they fail to appreciate the possibility of negative outcomes.

Previous studies have shown that lower insular activation has been shown to predict relapse in $\mathrm{MD}$ in decisionmaking tasks that did not involve risk (Clark et al, 2012; Paulus et al, 2005). Paulus et al (2005) showed that activation patterns in insula, posterior cingulate, middle frontal gyrus, and middle temporal gyrus all contributed to relapse prediction. Consistently, the present report shows differences in these same brain regions between MD who relapsed and those who remained abstinent. While the Paulus et al (2005) used a decision task, it did not involve risk. The present work suggests that risk-taking might be an important process that captures the salient features of likelihood for drug use and relapse since the decision to avoid uncertain, potentially dangerous situations require that an individual can distinguish between safe and risky alternatives. MD prone to relapse may fail to recruit the anterior insula to track contingencies in a decision-making task (ie, learning what options are risky $v s$ safe). Risk processing may be particularly important due to its role in evaluating uncertainty, which surrounds many decisions faced in daily life.

This study has several limitations. First, the follow-up data were collected by phone or in-person interview of the participants but not confirmed with urine analyses. However, we excluded individuals who met criteria for antisocial personality disorder to increase the reliability of self-report. Nevertheless, relapse status may have been under-reported. However, if under-reporting occurred, it suggests that fMRI results are relatively robust since they still detected significant effects. Second, we did not find any behavioral differences between the MD who relapsed and the MD who remained abstinent. There are several possible explanations for the lack of difference, including the relatively inconsequential nature of the task and the lack of an advantage between choosing risky vs safe options. If the safe option provided a net advantage across trials, then it may have helped to discriminate relapsing MD. We predict that in a more complex environment where the risk level of various options is more nuanced and rapidly changing, MD who relapse may be more likely to show behavioral changes (ie, riskier behavioral patterns) due to inefficient risk processing. Further, many neuroimaging studies show that brain activation is more sensitive to group differences than behavior (Rubia et al, 2009). Third, the low number of females recruited prevented a gender analysis and may limit the generalizability of these findings to females.

In summary, insula activation, in conjunction with other regions involved in risky decisions, during a risk-taking task can provide information that helps predict which MD are likely to relapse. Effectively evaluating risk may help individuals maintain abstinence for an extended period of time. Our results add to a growing literature demonstrating deficits in the neural processing and evaluation of negative affective cues in substance-dependent individuals. This further complements recent work suggesting that the anterior insula and associated interoceptive processes have a critical role in sustaining drug dependence (Naqvi and Bechara, 2010; Naqvi et al, 2007). Treatment providers may see improved success rates by helping patients properly frame decisions involving risk. 


\section{FUNDING AND DISCLOSURE}

The authors declare no conflict of interest.

\section{ACKNOWLEDGEMENTS}

We would like to thank Dr F Berger, T Flagan, H Donovan, D Leland, M Mortezaei and B Friedrich for assistance and support during data acquisition. This work was supported by grants from the National Institute on Drug Abuse (R01DA016663, P20-DA027834, R01-DA027797, and R01DA018307 as well as a VA Merit Grant to Martin Paulus).

\section{REFERENCES}

American Psychiatric Association (1994). Diagnostic and Statistical Manual of Mental Disorders. 4th edn. American Psychiatric Association: Washington, DC, pp 886.

Brown JW, Braver TS (2007). Risk prediction and aversion by anterior cingulate cortex. Cogn Affect Behav Neurosci 7: 266-277.

Clark VP, Beatty GK, Anderson RE, Kodituwakku P, Phillips JP, Lane TD et al (2012). Reduced fMRI activity predicts relapse in patients recovering from stimulant dependence. Hum Brain Mapp. doi:10.1002/hbm.22184.

Cousijn J, Wiers RW, Ridderinkhof KR, van den Brink W, Veltman DJ, Porrino LJ et al (2013). Individual differences in decision making and reward processing predict changes in cannabis use: a prospective functional magnetic resonance imaging study. Addict Biol 18: 1013-1023.

Cox RW (1996). AFNI: software for analysis and visualization of functional magnetic resonance neuroimages. Comput Biomed Res 29: 162-173.

Craig AD (2009). How do you feel-now? The anterior insula and human awareness. Nat Rev Neurosci 10: 59-70.

Ersche KD, Fletcher PC, Lewis SJ, Clark L, Stocks-Gee G, London $\mathrm{M}$ et al (2005). Abnormal frontal activations related to decisionmaking in current and former amphetamine and opiate dependent individuals. Psychopharmacology (Berl) 180: 612-623.

Fishbein DH, Eldreth DL, Hyde C, Matochik JA, London ED, Contoreggi C et al (2005). Risky decision making and the anterior cingulate cortex in abstinent drug abusers and nonusers. Brain Res Cogn Brain Res 23: 119-136.

Gowin JL, Mackey S, Paulus MP (2013). Altered risk-related processing in substance users: Imbalance of pain and gain. Drug Alcohol Depend 132: 13-21.

Harle KM, Chang LJ, van 't Wout M, Sanfey AG (2012). The neural mechanisms of affect infusion in social economic decisionmaking: a mediating role of the anterior insula. Neuroimage 61: 32-40.

Hesselbrock M, Easton C, Bucholz KK, Schuckit M, Hesselbrock V (1999). A validity study of the SSAGA-a comparison with the SCAN. Addiction 94: 1361-1370.

Kahneman D, Tversky A (1979). Prospect theory: an analysis of decision under risk. Econometrica 47: 263-291.

Kaufman JN, Ross TJ, Stein EA, Garavan H (2003). Cingulate hypoactivity in cocaine users during a GO-NOGO task as revealed by event-related functional magnetic resonance imaging. J Neurosci 23: 7839-7843.

Kim YT, Song HJ, Seo JH, Lee JJ, Lee J, Kwon DH et al (2011). The differences in neural network activity between methamphetamine abusers and healthy subjects performing an emotion-matching task: functional MRI study. NMR Biomed 24: $1392-1400$.

Koob GF (2008). A role for brain stress systems in addiction. Neuron 59: 11-34.
Kosten TR, Scanley BE, Tucker KA, Oliveto A, Prince C, Sinha R et al (2006). Cue-induced brain activity changes and relapse in cocaine-dependent patients. Neuropsychopharmacology 31: 644-650.

Kruschwitz JD, Simmons AN, Flagan T, Paulus MP (2012). Nothing to lose: processing blindness to potential losses drives thrill and adventure seekers. Neuroimage 59: 2850-2859.

Kuhnen CM, Knutson B (2005). The neural basis of financial risk taking. Neuron 47: 763-770.

Lancaster JL, Woldorff MG, Parsons LM, Liotti M, Freitas CS, Rainey L et al (2000). Automated Talairach atlas labels for functional brain mapping. Hum Brain Mapp 10: 120-131.

Li CS, Sinha R (2008). Inhibitory control and emotional stress regulation: neuroimaging evidence for frontal-limbic dysfunction in psycho-stimulant addiction. Neurosci Biobehav Rev 32: $581-597$.

Luo X, Zhang S, Hu S, Bednarski SR, Erdman E, Farr OM et al (2013). Error processing and gender-shared and -specific neural predictors of relapse in cocaine dependence. Brain 136(Pt 4): 1231-1244.

Marhe R, Luijten M, van de Wetering BJ, Smits M, Franken IH (2013). Individual differences in anterior cingulate activation associated with attentional bias predict cocaine use after treatment. Neuropsychopharmacology 38: 1085-1093.

McKetin R, Najman JM, Baker AL, Lubman DI, Dawe S, Ali R et al (2012). Evaluating the impact of community-based treatment options on methamphetamine use: findings from the Methamphetamine Treatment Evaluation Study (MATES). Addiction 107: 1998-2008.

Miller WR (1996). What is a relapse? Fifty ways to leave the wagon. Addiction 91(Suppl): S15-S27.

Naqvi NH, Bechara A (2010). The insula and drug addiction: an interoceptive view of pleasure, urges, and decision-making. Brain Struct Funct 214: 435-450.

Naqvi NH, Rudrauf D, Damasio H, Bechara A (2007). Damage to the insula disrupts addiction to cigarette smoking. Science 315: 531-534.

Nestor LJ, Ghahremani DG, Monterosso J, London ED (2011). Prefrontal hypoactivation during cognitive control in early abstinent methamphetamine-dependent subjects. Psychiatry Res 194: 287-295.

Paulus MP, Rogalsky C, Simmons A, Feinstein JS, Stein MB (2003). Increased activation in the right insula during risk-taking decision making is related to harm avoidance and neuroticism. Neuroimage 19: 1439-1448.

Paulus MP, Tapert SF, Schuckit MA (2005). Neural activation patterns of methamphetamine-dependent subjects during decision making predict relapse. Arch Gen Psychiatry 62: 761-768.

Payer DE, Lieberman MD, Monterosso JR, Xu J, Fong TW, London ED (2008). Differences in cortical activity between methamphetamine-dependent and healthy individuals performing a facial affect matching task. Drug Alcohol Depend 93: 93-102.

Preuschoff K, Quartz SR, Bossaerts P (2008). Human insula activation reflects risk prediction errors as well as risk. J Neurosci 28: 2745-2752.

Rosner B (2006). Fundamentals of Biostatistics. Thomson Brooks/ Cole: Belmont, CA, Vol 6: 863 pp.

Rubia K, Halari R, Christakou A, Taylor E (2009). Impulsiveness as a timing disturbance: neurocognitive abnormalities in attentiondeficit hyperactivity disorder during temporal processes and normalization with methylphenidate. Philos Trans $R$ Soc Lond B Biol Sci 364: 1919-1931.

Rudorf S, Preuschoff K, Weber B (2012). Neural correlates of anticipation risk reflect risk preferences. J Neurosci 32: $16683-16692$.

Schonberg T, Fox CR, Poldrack RA (2011). Mind the gap: bridging economic and naturalistic risk-taking with cognitive neuroscience. Trends Cogn Sci 15: 11-19. 
Seo D, Lacadie CM, Tuit K, Hong KI, Constable RT, Sinha R (2013). Disrupted ventromedial prefrontal function, alcohol craving, and subsequent relapse risk. JAMA Psychiatry 1-13.

Simmons AN, Arce E, Lovero KL, Stein MB, Paulus MP (2009). Subchronic SSRI administration reduces insula response during affective anticipation in healthy volunteers. Int J Neuropsychopharmacol 12: 1009-1020.
Slovic P, Finucane M, Peters E, MacGregor DG (2002). The affect heuristic. In: Gilovich TGriffin D (eds) Heuristics and Biases: The Psychology of Intuitive Judgment. Cambridge University Press: New York, NY, pp 397-420.

Uttl B (2002). North American Adult Reading Test: age norms, reliability, and validity. J Clin Exp Neuropsychol 24: $1123-1137$.

Supplementary Information accompanies the paper on the Neuropsychopharmacology website (http://www.nature.com/npp) 